\title{
Avaliaçáo in vitro do fungo predador de nematoides Duddingtonia flagrans sobre larvas infectantes de ciatostomíneos de equinos (Nematoda: Cyathostominae)
}

\author{
In vitro evaluation of nematode predacious fungus Duddingtonia flagrans on cyathostomes \\ infective larvae of equines (Nematoda: Cyathostominae)
}

Fabio R. Braga; Jackson V. Araújo*; Juliana M. Araujo; André R. Silva; Rogério O. Carvalho; Artur K. Campos

Departamento de Medicina Veterinária, Universidade Federal de Viçosa - UFV

Recebido em 29 de Novembro de 2007

Aceito em 18 de Maio de 2009

\section{Resumo}

A capacidade predatória de um isolado de fungo predador de nematoides Duddingtonia flagrans (AC001) sobre larvas infectantes de ciatostomíneos foi avaliada em condições laboratoriais em ensaio experimental em meio ágar-água $2 \%$ (AA 2\%). Houve redução significativa $(\mathrm{p}<0,01)$ de $93,64 \%$ na média de larvas infectantes de ciatostomíneos recuperadas do meio AA2\%, ao final de sete dias. Os resultados desse ensaio evidenciam que o isolado fúngico AC001 poderia ser utilizado no controle biológico de ciatostomíneos de equinos.

Palavras-chave: Controle biológico, Strongyloidea, fungos nematófagos, fungos predadores de nematoides, controle alternativo.

\begin{abstract}
The predatory capacity of one isolate of nematode-trapping fungus Duddingtonia flagrans (AC001) on infective larvae of cyathostomes was evaluated in laboratorial conditions in medium water-agar 2\% (WA 2\%). There was significant reduction ( $\mathrm{p}<0.01)$ of $93.64 \%$ in the average of infective larvae of cyathostomes recovered of medium WA $2 \%$ at seven day. These results show that the isolated AC001 could be used in the biological control of cyathostomes of horses.
\end{abstract}

Keywords: Biological control, Strongyloidea, nematophagous fungi, nematode-trapping fungi, alternative control.

Nematoides estrongilídeos são comuns em equinos, representando um grupo de grande importância no Brasil, já que grande parte do rebanho encontra-se infectado. A subfamília Cyathostominae é a mais prevalente com registros de animais parasitados com mais de um milhão e duzentas mil espécimes de ciatostomíneos, que causam prejuízo à saúde animal (CASTRO et al., 2003; ANJOS; RODRIGUES, 2006).

Resultados de pesquisas realizadas a campo sugerem que os equinos adquirem resistência aos pequenos estrongilídeos com a idade, verificados através da redução da carga parasitária e a contagem de ovos nas fezes. Essa resposta, porém, é lenta e inconsistente na maioria dos animais e náo tem relaçáo com a intensidade do contato parasitário anterior (ASSIS; ARAÚJO, 2003; ANJOS; RODRIGUES, 2006).

Os fungos nematófagos constituem uma opçáo ao controle dos nematoides gastrintestinais de animais domésticos. Sua ação está

\footnotetext{
*Autor para correspondência: Jackson V. Araújo

Departamento de Medicina Veterinária, Universidade Federal de Viçosa - UFV,

Avenida Peter Henry Rolfs s/n, Campus Universitário, CEP 36570-000

Viçosa - MG, Brasil; e-mail: jvictor@ufv.br

Apoio: Este projeto foi financiado pelo CNPq, CAPES e FAPEMIG.
}

concentrada no ambiente fecal e direcionada ao combate das larvas de vida livre dos parasitos (CASTRO et al., 2003; ARAÚJO et al., 2004). Eles são cosmopolitas, ocorrem em solos naturais, solos agricultáveis e em todos os tipos de matéria orgânica em decomposição (ARAÚJO et al., 2006). Duddingtonia flagrans é a espécie do grupo de fungos predadores, mais promissora no controle das helmintoses gastrintestinais de animais domésticos devido à produção de clamidósporos em abundância (MOTA et al., 2003).

$\mathrm{O}$ presente estudo objetivou avaliar a capacidade predatória in vitro do fungo $D$. flagrans (isolado AC001) oriundo de solo do Brasil, na localidade de Viçosa, na zona da mata de Minas Gerais, sobre larvas infectantes de ciatostomíneos.

Panagrellus sp. (nematoides de vida livre) foram mantidos em placas de Petri com meio de aveia em flocos, umedecida e amassada. Esses nematoides foram extraídos do meio de cultura através da imersão de pequenas quantidades de aveia em água destilada à temperatura ambiente, no aparelho de Baermann, e coletados em tubos de hemólise após seis horas de decantação.

Discos de cultura de $4 \mathrm{~mm}$ de diâmetro foram extraídos dos isolados fúngicos mantidos em tubos de ensaio, contendo "corn meal agar" $2 \%$, e transferidos para placas de Petri de 9,0 cm de 
diâmetro, com $20 \mathrm{~mL}$ de batata-dextrose-ágar 2\%, e mantidos a $25{ }^{\circ} \mathrm{C}$, no escuro, durante 10 dias. Após o crescimento dos isolados, novos discos de cultura de $4 \mathrm{~mm}$ de diâmetro foram transferidos para placas de Petri de $9,0 \mathrm{~cm}$ de diâmetro, contendo $20 \mathrm{~mL}$ de ágar-água 2\% (AA 2\%). Nesse meio, foi acrescentado, diariamente, $1 \mathrm{~mL}$ de água destilada contendo 1.000 larvas de Panagrellus sp., durante um período de 21 dias, para indução de formação de conídios fúngicos. Quando se observou o completo desenvolvimento fúngico, $5 \mathrm{~mL}$ de água destilada foram adicionados a cada placa de Petri. Os conídios e fragmentos miceliais foram removidos segundo a técnica descrita de Araújo et al. (1993).

Fezes frescas foram coletadas diretamente da ampola retal de 16 equinos (Equus caballus) mestiços, com idade entre 3-8 anos, naturalmente infectados. A seguir, realizou-se a contagem de ovos por grama de fezes (OPG) de acordo com Gordon e Withlock (1939), com a finalidade de encontrar animais positivos. Posteriormente, procedeu-se a realização das coproculturas e, ao final de sete dias, foram obtidas larvas de terceiro estádio $\left(\mathrm{L}_{3}\right)$, que foram identificadas e quantificadas segundo os critérios descritos por Bevilaqua et al. (1993), em microscópio óptico, objetiva de 10x. A leitura do Baermann demonstrou que $100 \%$ das $\mathrm{L}_{3}$ visualizadas eram de ciatostomíneos.

Dois grupos foram formados em placas de Petri de 9,0 cm de diâmetro, contendo $20 \mathrm{~mL}$ de AA2\%, um grupo tratado e um grupo controle, sendo feitas 10 repetiçôes para cada grupo. As placas de Petri foram previamente marcadas em campos de $4 \mathrm{~mm}$ de diâmetro. No grupo tratado, cada placa de Petri continha $1.000 \mathrm{~L}_{3}$ de ciatostomíneos e 1.000 conídios do isolado fúngico AC001 em AA2\%; e o grupo controle (sem isolado fúngico) continha apenas $1.000 \mathrm{~L}_{3}$ nas placas com AA2\%. Durante sete dias, a cada 24 horas, 10 campos aleatórios de $4 \mathrm{~mm}$ de diâmetro em cada placa dos grupos tratado e controle foram observados em microscópio óptico em objetiva de $10 \times$, contando-se o número de $\mathrm{L}_{3}$ não predadas em cada um. No sétimo dia, foram recuperadas as $\mathrm{L}_{3}$ não predadas do conteúdo das placas de Petri, por meio do aparelho de Baermann, com água a $42{ }^{\circ} \mathrm{C}$. A média de $\mathrm{L}_{3}$ de ciatostomíneos recuperadas foi calculada. Os dados obtidos foram transformados em $\log (\mathrm{x}+1) \mathrm{e}$, posteriormente, submetidos às análises de variância (ANOVA) em níveis de significância de 1 e $5 \%$ de probabilidade. A eficiência de predação de $\mathrm{L}_{3}$ em relação ao controle foi avaliada pelo teste de Tukey ao nível de $1 \%$ de probabilidade. Posteriormente, o percentual de redução da média de $\mathrm{L}_{3}$ foi calculado, utilizando-se a Equação 1:

$\%$ Reduçáo $=\underline{\left(\text { Média de } \mathrm{L}_{3} \text { recuperadas do controle }- \text { Média de } \mathrm{L}_{3} \text { recuperadas do tratamento }\right)} \times 100(1)$ Média de $\mathrm{L}_{3}$ recuperadas do controle

O isolado AC001 do fungo D. flagrans foi capaz de predar as larvas de ciatostomíneos ao longo do ensaio realizado in vitro. Essa predação foi visualizada nas placas de Petri do grupo tratado já na primeira leitura dos campos, realizada no período de 24 horas após a interação das larvas com o isolado fúngico. Contudo não foi observada a presença do fungo e táo pouco de armadilhas formadas predando as $\mathrm{L}_{3}$ presentes na placas de Petri do grupo controle.

A comprovação dessa predação foi observada com a redução das médias de $\mathrm{L}_{3}$ recuperadas de ciatostomíneos, ao final do ensaio in vitro, no sétimo dia (Figura 1). Ao final de sete dias, o isolado AC001 demonstrou melhor desempenho em relação ao controle, observando-se uma redução significativa $(\mathrm{p}<0,01)$ de 93,64\% no número médio das $\mathrm{L}_{3}$ recuperadas por meio do aparelho de Baermann.

A média de larvas infectantes de $\mathrm{L}_{3}$ não predadas de ciatostomíneos por campo de $4 \mathrm{~mm}$ de diâmetro durante o experimento, está representada na Tabela 1 . De acordo com Fontenot et al. (2003), D. flagrans tem uma taxa ótima de crescimento em temperatura entre 20 e $30{ }^{\circ} \mathrm{C}$ de 15 e $60 \mathrm{~mm}$ por semana. $\mathrm{Na}$ presença de nematoides, esse fungo consegue produzir de 700 a 800 armadilhas por $\mathrm{cm}^{2}$ em curto intervalo de tempo. Santos et al. (2001) registraram a eficácia de duas espécies de fungos nematófagos e, dentre essas, D. flagrans em fezes de equinos naturalmente infectados por ciatostomíneos,

Tabela 1. Média diária e desvio-padrão de larvas infectantes $\left(\mathrm{L}_{3}\right)$ não predadas de ciatostomíneos por campo de $4 \mathrm{~mm}$ de diâmetro, em meio ágar-água $2 \%$, durante o período de 7 dias nos tratamentos com isolado fúngico de Duddingtonia flagrans (AC001) e no controle sem fungo.

\begin{tabular}{ccc}
\hline \multirow{2}{*}{$\begin{array}{c}\text { Tempo } \\
\text { (dias) }\end{array}$} & \multicolumn{2}{c}{ Tratamento $^{\text {(média de } \mathbf{L}_{\mathbf{3}} \text { náo predadas } \pm \text { dp) }}$} \\
\cline { 2 - 3 } & AC001 & Controle \\
\hline 1 & $1,8 \pm 1,66^{\mathrm{A}}$ & $3,05 \pm 4,77^{\mathrm{B}}$ \\
2 & $0,84 \pm 0,80^{\mathrm{A}}$ & $2,09 \pm 1,08^{\mathrm{B}}$ \\
3 & $0,73 \pm 0,92^{\mathrm{A}}$ & $2,26 \pm 1,51^{\mathrm{B}}$ \\
4 & $0,86 \pm 1,08^{\mathrm{A}}$ & $2,51 \pm 1,99^{\mathrm{B}}$ \\
5 & $0,39 \pm 0,85^{\mathrm{A}}$ & $2,29 \pm 1,49^{\mathrm{B}}$ \\
6 & $0,45 \pm 0,83^{\mathrm{A}}$ & $2,96 \pm 2,84^{\mathrm{B}}$ \\
7 & $0,20 \pm 0,38^{\mathrm{A}}$ & $2,97 \pm 2,24^{\mathrm{B}}$ \\
\hline
\end{tabular}

Médias seguidas pela mesma letra maiúscula $(\mathrm{A} ; \mathrm{B})$ nas linhas não diferem estatisticamente a $1 \%$ de probabilidade do teste de Tukey.

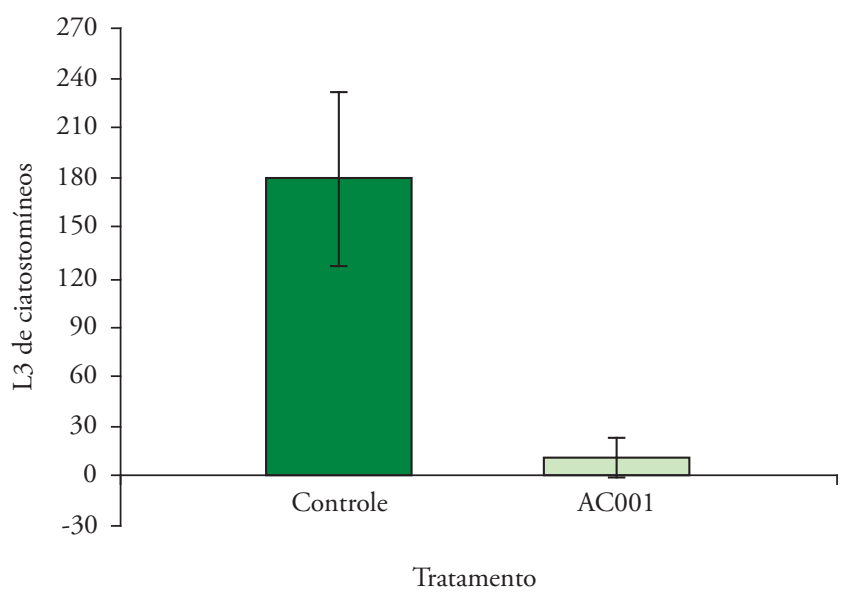

Figura 1. Média de larvas infectantes $\left(\mathrm{L}_{3}\right)$ de ciatostomíneos não predadas, recuperadas em meio ágar-água $2 \%$ pelo método de Baermann, no sétimo dia do tratamento, após interaçáo com isolado fúngico Duddingtonia flagrans (AC001) e do controle sem fungos. Barra representa o desvio-padrão. 
observando-se que $D$. flagrans, quando estimulado em temperatura de 10 a $20^{\circ} \mathrm{C}$, apresentou percentuais de reduçáo larvar em torno de 47,5 e 41,8\% após 14 dias em coprocultura. Durante o presente trabalho, a temperatura foi mantida constante na faixa de $28{ }^{\circ} \mathrm{C}$, e foi observado que houve uma redução eficaz do número de $\mathrm{L}_{3}$ de ciatostomíneos pela presença do fungo $D$. flagrans após sete dias de interação.

Assis e Araújo (2003), trabalhando com Monacrosporium sinense e $M$. appendiculatum, notaram que, ao final dos 15 dias, houve uma redução do número médio de larvas infectantes de ciatostomíneos recuperadas das placas e das coproculturas em torno de $70 \%$, quando comparado ao grupo controle $(\mathrm{p}<0,05)$. Os resultados obtidos no presente trabalho sugerem que o fungo D. flagrans é mais eficaz que essas duas espécies de fungos sobre $\mathrm{L}_{3}$ de ciatostomíneos ao final de sete dias.

$\mathrm{O}$ isolado testado de D. flagrans foi eficaz no controle in vitro de larvas infectantes de ciatostomíneos de equinos e poderia ser utilizado no controle biológico desses nematoides.

\section{Referências}

ANJOS, D. H. S.; RODRIGUES, M. L. A. Diversity of the infra communities of strongylid nematodes in the ventral colon of Equus caballus from Rio de Janeiro state, Brazil. Veterinary Parasitology, v. 136, n. 3-4, p. 251-257, 2006.

ARAÚJO, J. V. et al. Antagonistic effect of predacious Arthrobotrys fungi on infective Haemonchus placei larvae. Journal of Helminthology, v. 67, n. 3, p. 136-138, 1993.

ARAÚJO, J. V.; MOTA, M. A.; CAMPOS, A. K. Controle biológico de helmintos parasitos de animais por fungos nematófagos. Revista Brasileira de Parasitologia Veterinária, v. 13, supl. 1, p. 165-170, 2004.
ARAÚJO, J. V. et al. Avaliação do fungo predador de nematoides Duddingtonia flagrans sobre larvas infectantes de Haemonchus contortus e Strongyloides papillosus de caprinos. Revista Brasileira de Parasitologia Veterinária, v. 15, n. 2, p. 76-79, 2006.

ASSIS, R. C. L.; ARAÚJO J. V. Avaliação da viabilidade de duas espécies de fungos predadores do gênero Monacrosporium sobre ciatostomíneos após a passagem pelo trato gastrintestinal de eqüinos em formulação de alginato de sódio. Revista Brasileira de Parasitologia Veterinária, v. 12, n. 3, p. 109-113, 2003.

BEVILAQUA, C. M. L.; RODRIGUES, M. L.; CONCORDET, D. Identification of infective larvae of some common nematode strongylids of horses. Revue Médicine Véterinaire, v. 144, n. 12, p. 989-995, 1993.

CASTRO, A. A. et al. Potencial dos fungos nematófagos Arthrobotrys sp. e Monacrosporium thaumasium para o controle de larvas de ciatostomíneos de eqüinos (Nematoda: Cyathostominae). Revista Brasileira de Parasitologia Veterinária, v. 12, n. 2, p. 53-57, 2003.

FONTENOT, M. E. et al. Efficiency of feeding Duddingtonia flagrans chlamydospores to grazing ewes on reducing availability of parasitic nematode larvae on pasture. Veterinary Parasitology, v. 118, n. 3-4, p. 203-213, 2003.

GORDON, H. M.; WHITLOCK, H. V. A new technique for counting nematode eggs in sheep faeces. Journal of the Council for Scientific Industrial Research, v. 12, p. 50-52, 1939.

MOTA, M. A.; CAMPOS, A. K.; ARAÚJO, J. V. Controle biológico de helmintos parasitos de animais: estágio atual e perspectivas futuras. Pesquisa Veterinária Brasileira, v. 23, n. 3, p. 93-100, 2003.

SANTOS, C. P.; PADILHA, T.; RODRIGUES, M. L. A. Predatory activity of Arthrobotrys oligospora and Duddingtonia flagrans on pre-parasitic larval stages of cyathostominae under different constant temperatures. Ciência Rural, v. 31, n. 5, p. 839-842, 2001. 